\title{
AVALIAÇÃO DE CARACTERES VEGETATIVOS DE HÍBRIDOS DE COQUEIRO (COCOS NUCIFERA L.) NA REGIÃO NÃO PANTANOSA DO MUNICÍPIO DE POCONÉ, MT. ${ }^{1}$
}

\author{
HUMBERTO DE CARVALHO MARCILIO ${ }^{2}$, HILTON NEY GAÍVA³ , JOADIL GONÇALVES DE ABREU, \\ WILSON MENEZES ARAGÃO ${ }^{5}$ E JOSÉ CARLOS FRESCHI ${ }^{6}$.
}

\begin{abstract}
RESUMO - O presente estudo foi realizado entre março de 1999 e março de 2000, na região de Cangas, município de Poconé, MT, com a finalidade de estudar o desenvolvimento inicial de híbridos intervarietais de coqueiros, avaliados para as características: circunferência do coleto (CC), número de folhas vivas (NFV), número de folhas emitidas (NFE), número de folíolos da folha 3 (NFoF3), comprimento de folíolos da folha3 (CFoF3), comprimento da folha 3 (CF3) e comprimento do pecíolo da folha 3 (CPF3). Utilizou-se o delineamento experimental em blocos casualizados, com quatro repetições e cinco tratamentos, tendo em cada parcela 16 plantas úteis. Os resultados das análises estatísticas demonstraram haver diferença significativa para todas as características. O híbrido AVG x GBrPF foi o que melhor expressou o "vigor híbrido" com maiores CC, NFoF3, CFoF3 e CF3. Por outro lado, o AVeJ x GBrRN apresentou os menores valores para estas características, embora tenha expressado o melhor resultado para NFE e NFV nas avaliações finais.
\end{abstract}

Termos para indexação: coqueiro, híbridos, melhoramento.

\section{VEGETATIVE COCONUT PALM HYBRID (Cocos nucifera L.) BEHAVIOR ON NON MARSHY AREA OF POCONÉ (MT-BRAZIL)}

\begin{abstract}
This study was accomplished form March, 1999 up to march 2000, in Cangas, Poconé, Mato Grosso, Brazil. It aimed studying the initial development of hybrid coconut palms (Dwarf x Tall), by evaluating the characteristics: girth circunference (CC); live number of leaves (NFV); emitted leaves number of (NFE); number of leaflets at leaf 3 (NFoF3); leaflets length at leaf 3 (NFoF3); leaf 3 length (CF3) and petiole length at leaf 3 (CPF3). The experimental design used was casual blocks with four repetitions and five treatments, each plot had 16 useful plants. The statistical analysis results showed a significant difference for all characteristics. The AVG x GBrPF hybrid showed the best "hybrid vigor" with greater CC, NFoF3, CFoF3 and CF3. On the other hand, the AVeJ x GBrRN hybrid showed the smallest values for these characteristics, although it has expressed best result for NFE and NFV at final evaluations.
\end{abstract}

Index terms: Hybrid, coconut palm, breeding.

O coqueiro (Cocos nucifera L.) é considerado a espécie tropical de maior importância socioeconômica das regiões intertropicais, devido à versatilidade do uso da planta. Tem um grande papel social, principalmente nas regiões costeiras, onde é cultivado, em sua grande maioria, por pequenos produtores, em solos arenosos e pobres, sem aptidão para outro tipo de atividade. Sendo também considerado uma das mais importantes oleaginosas do mundo, ocupando a quinta posição na produção mundial de óleos vegetais (Persley, 1992).

A área plantada e a produção brasileira de coco, entre os principais países produtores, são relativamente pequenas, embora tenham aumentado nos últimos anos, chegando a mais de 260 mil hectares em 1997, representando menos de $2 \%$ do que é produzido mundialmente. A produção é voltada principalmente para o mercado interno, e a maior concentração de área plantada ainda se encontra na região Nordeste do Brasil (Pires et al., 1999).

Verifica-se, no entanto, rápido crescimento nas áreas de plantio nas outras regiões do País, ocasionado principalmente pelo aumento do consumo de água de coco, associado aos preços elevados obtidos pelos produtores.

No Brasil, a expansão da área de plantio com o coqueiro vem ocorrendo principalmente com os anões verdes, destinados, principalmente, para o consumo de água, embora esteja ocorrendo novas áreas, com plantio de híbridos intervarietais anão x gigante,

1 Trabalho $n^{\circ}$ 083/2000. Recebido: 05/05/2000. Aceito para publicação: 03/05/2001. Parte de dissertação de mestrado do $1^{\circ}$ autor. Apoio Financeiro FAPEMAT / CNPq

2 Eng. Agr. EMPAER-MT. Mestrando em Agricultura Tropical, FAMEV/UFMT. Cuiabá, MT. E-mail: hcmbeto@uol.com.br

3 Eng. Agr., Dr., Professor Adjunto. Depto. de Fitotecnia e Fitossanidade. FAMEV/UFMT, Av. Fernando Corrêa da Costa, S/Nº, Cidade Universitária, 78060-900, Cuiabá, MT.

4 Eng. Agr., M.Sc., Professor Assistente. Depto. de Zootecnia e Extensão Rural. FAMEV/UFMT, Av. Fernando Corrêa da Costa, S/Nº Cidade Universitária, 78060-900, Cuiabá, MT.

5 Eng. Agr., Dr., Embrapa Tabuleiros Costeiros, Cx.P.44, CEP 49001-970, Aracajú, SE.

6. Estudante de agronomia, FAMEV/UFMT, Bolsista PIBIC/CNPq. 
que apresenta a vantagem de ser utilizado tanto na forma "in natura" (uso doméstico e água de coco) como nas agroindústrias (leite de coco, etc.). Situação semelhante pode ser verificada, também, no Estado de Mato Grosso, cuja área plantada se situa ao redor de 3.500 hectares, representando $1,5 \%$ da área total de plantio no Brasil (Produção Agrícola Municipal, 1998).

Mesmo com a grande expansão da cultura, a produtividade ainda é extremamente baixa, provocada principalmente pela utilização de cultivares não selecionadas, associada ao manejo inadequado.

O presente estudo objetivou avaliar o desenvolvimento inicial entre híbridos de coqueiros-anões e gigantes, nas condições da região não pantanosa de Poconé, Estado de Mato Grosso.

O trabalho foi desenvolvido na Granja Água Viva, distrito de Cangas (latitude Sul $16^{\circ} 15^{\prime} 24^{\prime \prime}$ e longitude W.GR 56 $36^{\circ}$ 24"), município de Poconé-MT, em uma área de LATOSSOLO VERMELHO-AMARELO Eutrófico (Embrapa, 1999).

O clima da região é classificado como tropical quente e subúmido (Ferreira, 1997), com duas estações bem definidas, um período seco, de abril a setembro, e outro chuvoso, de outubro a março (Figura 1). As mudas foram cedidas pela Embrapa / CPATC, Aracajú - SE, e plantadas utilizando-se do delineamento experimental em blocos casualizados, com cinco tratamentos (híbridos) e quatro repetições. Os híbridos avaliados foram: T1 - Anão amarelo de Gramame (AAG) x Gigante do Brasil da Praia do Forte (GBrPF);

T2 - Anão vermelho de Gramame (AVG) x Gigante do Brasil da Praia do Forte (GBrPF);

T3 - Anão amarelo de Gramame (AAG) x Gigante do Oeste Africano (GOA);

T4 - Anão vermelho de Gramame (AVG) x Gigante do Oeste Africano (GOA);

T5 - Anão verde de Jiqui (AVeJ) x Gigante do Brasil do Rio Grande do Norte (GBrRN).

Foram avaliadas 16 plantas úteis por parcela, plantadas no espaçamento de $8,5 \mathrm{~m}$ em triângulo equilátero, numa densidade de 160 plantas por hectare. A área experimental contou com uma bordadura externa constituída por coqueiros híbridos.

As avaliações foram realizadas em intervalos regulares de seis meses, de março de 1999 a março de 2000. Estudaram-se as seguintes características: circunferência do coleto (CC); número de folhas vivas (NFV); número de folhas emitidas (NFE); número de folíolos na folha três (NFoF3); comprimento do folíolo na folha três (CFoF3); comprimento da folha três (CF3); comprimento do pecíolo da folha três (CPF3). Foram realizadas análises de variância para todas as características, utilizando-se do teste de Tukey a 5\% de probalidade, para comparação de médias.

Nas Tabelas 1, 2 e 3, podem-se visualizar os valores médios obtidos para: CC e NFV; NFE e NFoF3; CFoF3, CF3 e CPF3, respectivamente, aos 14; 20 e 26 meses após o plantio.

Nota-se, na Tabela 1, que o AVG x GBrPF apresentou maior circunferência do coleto. Estes resultados indicam um bom desenvolvimento vegetativo quando comparados aos obtidos por Siqueira et al. (1995), com híbridos, em São Cristóvão e Pirambu-SE, aos 39 meses $(147,4 \mathrm{~cm}$ para o $\mathrm{PB}-213$ e $107,9 \mathrm{~cm}$ para os PB-141 e PB-111), e aos valores obtidos por Ferraz et al. (1987) em Goiana-PE, aos 19 meses (55cm para o PB-122 e $66 \mathrm{~cm}$ para o PB-111), e Ouriver (1984), na Costa do Marfim, com híbrido PB121 (AAM x GOA) aos 16 e 28 meses, quando foi constatada uma circunferência de coleto de $36 \mathrm{e} 115 \mathrm{~cm}$, respectivamente.

As médias obtidas no vigésimo mês, para o número de folhas vivas, variaram de 8,28 a 9,42, cujo desenvolvimento vegetativo assemelha-se ao dos híbridos estudados por Ferraz et al.(1987), que apresentaram valores entre 9,7 para os híbridos PB-113 e PB-121 e 8,0 para o híbrido PB-213, aos 19 meses após o plantio.

A maior taxa de produção anual de folhas foi verificada para o híbrido AVeJ x GBrRN (12,16 folhas/ano). Por outro lado, o AVG x GOA apresentou a menor taxa (9,86 folhas/ano), estando de acordo com os resultados observados por Santos et al. (1982), no segundo ano de observação, nas Filipinas, com os híbridos PB-121 (12,44 folhas/ano) e PB-141 (11,4 folhas/ano), indicando bom comportamento dos híbridos avaliados, nas condições do experimento.

Em geral, os híbridos apresentaram uma boa performance com relação ao número de folíolos na folha 3 , quando comparados aos resultados obtidos por Ferraz et al. (1987), que encontraram 112 folíolos para PB-111 e 98 para o PB-121, aos 19 meses.

De acordo com a Tabela 3, o AVG x GBrPF apresentou os maiores comprimentos de folíolos da folha 3 e folhas 3 mais compridas, diferindo estatisticamente do AVeJ x GBrRN, nos períodos avaliados. Os resultados obtidos neste trabalho, para

TABELA 1 - Média dos dados de circunferência do coleto (CC, em $\mathrm{cm}$ ) e número de folhas vivas (NFV), avaliados em híbridos intervarietais do coqueiro, no período de 1999/2000. Poconé - MT, 2000.

\begin{tabular}{|c|c|c|c|c|c|c|c|c|c|}
\hline \multirow{4}{*}{$\begin{array}{l}\text { TR A T A M ENTOS } \\
\text { (HÍB RIDOS) }\end{array}$} & \multicolumn{9}{|c|}{ Período $^{1}$} \\
\hline & $\mathrm{M}$ arço & 199 & Set $/ 9$ & & Março & 00 & Março / 99 & Set / 99 & $\mathrm{M}$ arço / 00 \\
\hline & $14 \mathrm{me}$ & ses & $20 \mathrm{mes}$ & & $26 \mathrm{mes}$ & & 14 meses & 20 meses & 26 meses \\
\hline & \multicolumn{6}{|c|}{ Circunferência do coleto (CC) } & \multicolumn{3}{|c|}{ Número de folhas vivas (NFV) } \\
\hline $\mathrm{AVG} \times \mathrm{GBrPF}$ & 54,88 & $\bar{a}$ & 78,47 & $\bar{a}$ & 122,64 & $\mathbf{a}$ & 8,25 & $9,00 \quad \mathrm{ab}$ & 12,52 \\
\hline$A A G \times G B r P F$ & 46,47 & $a b$ & 72,67 & $a b$ & 114,92 & $\mathrm{a}$ & 8,27 & 9,42 a & 12,09 \\
\hline AVG $x$ GOA & 46,38 & $a b$ & 71,65 & $\mathrm{ab}$ & 117,31 & $\mathrm{a}$ & 7,98 & 8,28 & 11,43 \\
\hline AAG $x$ GOA & 42,72 & $b c$ & 69,40 & $a b$ & 115,74 & $\mathrm{a}$ & 8,14 & 9,33 a & 12,14 \\
\hline A VeJ $x$ GBrRN & 33,84 & $\mathrm{c}$ & 59,53 & $\mathrm{~b}$ & 91,71 & $\mathrm{~b}$ & 7,44 & $8,94 \mathrm{ab}$ & 13,02 \\
\hline Média & 44,86 & & 70,34 & & 112,46 & & 8,02 & 8,99 & 12,24 \\
\hline $\mathrm{CV}(\%)$ & 10,24 & & 8,63 & & 5,89 & & 5,19 & 4,85 & 7,76 \\
\hline Teste de F & ** & & * & & ** & & & $*$ & \\
\hline
\end{tabular}

1 - Médias seguidas pela mesma letra na coluna não diferem significativamente entre si, ao nível de $5 \%$ de probabilidade, pelo teste de Tukey.

${ }^{*} ;{ }^{* *}$ - significativo aos níveis de 5 e $1 \%$ de probabilidade, pelo teste $\mathrm{F}$. 
TABELA 2 - Média dos dados de número de folhas emitidas (NFE) e número de folíolos da folha 3 (NFoF3), avaliados em híbridos intervarietais do coqueiro, no período de 1999 / 2000. Poconé - MT, 2000.

\begin{tabular}{|c|c|c|c|c|c|c|c|c|}
\hline \multirow{4}{*}{$\begin{array}{l}\text { TRATAMENTOS } \\
\text { (HÍBRIDOS) }\end{array}$} & \multicolumn{8}{|c|}{ Período $^{1}$} \\
\hline & Março / 99 & Set $/ 99$ & & Março / & & Março / 99 & Set $/ 99$ & Março / 00 \\
\hline & 14 meses & 20 mese & & 26 mese & & 14 meses & 20 meses & 26 meses \\
\hline & \multicolumn{5}{|c|}{ Número de folhas emitidas (NFE) } & \multicolumn{3}{|c|}{ Número de folíolos na folha 3 (NFoF3) } \\
\hline AVeJ x GBrRN & 1,87 & 1,80 & a & 3,06 & $\mathrm{a}$ & $72,99 \quad a b$ & 90,67 & $137,95 \mathrm{~b}$ \\
\hline AAG $x$ GBrPF & 1,84 & 1,45 & $a b$ & 2,55 & $\mathrm{~b}$ & 76,79 ab & $101,47 \mathrm{ab}$ & 156,03 a \\
\hline AAG $x$ GOA & 1,90 & 1,38 & $a b$ & 2,78 & $a b$ & 72,68 ab & $95,31 \quad b$ & 153,84 a \\
\hline$A V G \times G B r P F$ & 1,70 & 1,30 & $\mathrm{~b}$ & 2,66 & $a b$ & 80,85 a & 109,34 a & 164,74 a \\
\hline AVG $x$ GOA & 1,62 & 1,31 & $\mathrm{~b}$ & 2,56 & $\mathrm{~b}$ & $68,43 \mathrm{~b}$ & $102,23 \mathrm{ab}$ & 160,75 a \\
\hline Média & 1,79 & 1,45 & & 2,72 & & 74,35 & 99,80 & 154,66 \\
\hline CV (\%) & 7,70 & 14,00 & & 7,88 & & 5,79 & 6,16 & 4,14 \\
\hline Teste F & & * & & * & & * & * & ** \\
\hline
\end{tabular}

1 - Médias seguidas pela mesma letra na coluna não diferem significativamente entre si, ao nível de $5 \%$ de probabilidade, pelo teste de Tukey.

*;** - significativo aos níveis de 5 e $1 \%$ de probabilidade, pelo teste $\mathrm{F}$.

TABELA 3 - Média dos dados de comprimento de folíolos da folha 3 (CFoF3), comprimento da folha 3 (CF3) e comprimento do pecíolo da folha 3 (CPF3), avaliados em híbridos intervarietais do coqueiro, no período de 1999/00. Poconé - MT, 2000.

\begin{tabular}{|c|c|c|c|c|c|c|c|c|c|c|c|c|}
\hline \multirow{4}{*}{$\begin{array}{l}\text { TRATAMENTOS } \\
\text { (HÍBRIDOS) }\end{array}$} & \multicolumn{12}{|c|}{ Período $^{\top}$} \\
\hline & \multirow{2}{*}{\multicolumn{2}{|c|}{$\begin{array}{c}\text { Set / } 99 \\
20 \text { meses }\end{array}$}} & \multirow{2}{*}{\multicolumn{2}{|c|}{$\begin{array}{c}\text { Março / } 00 \\
26 \text { meses }\end{array}$}} & \multirow{2}{*}{\multicolumn{2}{|c|}{$\begin{array}{c}\text { Set / } 99 \\
20 \text { meses }\end{array}$}} & \multirow{2}{*}{\multicolumn{2}{|c|}{$\begin{array}{c}\text { Março / } 00 \\
26 \text { meses }\end{array}$}} & \multirow{2}{*}{\multicolumn{2}{|c|}{$\begin{array}{c}\text { Set / } 99 \\
20 \text { meses }\end{array}$}} & \multirow{2}{*}{\multicolumn{2}{|c|}{$\begin{array}{c}\text { Março / } 00 \\
26 \text { meses }\end{array}$}} \\
\hline & & & & & & & & & & & & \\
\hline & \multicolumn{4}{|c|}{ CFoF3 } & \multicolumn{4}{|c|}{ CF3 } & \multicolumn{4}{|c|}{ CPF3 } \\
\hline AAG $\times$ GBrPF & 88,55 & $\mathrm{a}$ & 107,53 & $a b$ & 237,39 & $a b$ & 362,01 & $\mathrm{a}$ & 50,96 & $\mathrm{a}$ & 63,18 & $\mathrm{a}$ \\
\hline AVG $x$ GBrPF & 96,27 & $\mathrm{a}$ & 113,03 & $\mathrm{a}$ & 260,19 & $\mathrm{a}$ & 384,14 & $\mathrm{a}$ & 49,07 & $\mathrm{a}$ & 57,86 & $\mathrm{a}$ \\
\hline $\mathrm{AAG} \times \mathrm{GOA}$ & 88,26 & $\mathrm{a}$ & 105,35 & $a b$ & 220,76 & b & 350,75 & $\mathrm{a}$ & 48,30 & $\mathrm{a}$ & 62,02 & $\mathrm{a}$ \\
\hline $\mathrm{AVG} \times \mathrm{GOA}$ & 88,98 & $\mathrm{a}$ & 109,16 & $a b$ & 240,87 & $a b$ & 366,76 & $\mathrm{a}$ & 49,32 & $\mathrm{a}$ & 62,62 & $\mathrm{a}$ \\
\hline AVeJ x GBrRN & 75,21 & $\underline{b}$ & 99,68 & $\mathrm{~b}$ & 177,31 & c & 259,76 & $\underline{b}$ & 35,79 & $\mathrm{~b}$ & 37,76 & $\mathrm{~b}$ \\
\hline Média & 87,45 & & 106,95 & & 227,30 & & 344,68 & & 46,69 & & 56,69 & \\
\hline CV (\%) & 6,41 & & 4,73 & & 7,51 & & 5,34 & & 4,13 & & 4,45 & \\
\hline Teste F & ** & & * & & ** & & $* *$ & & $* *$ & & ** & \\
\hline
\end{tabular}

1 - Médias seguidas pela mesma letra na coluna não diferem significativamente entre si, ao nível de $5 \%$ de probabilidade, pelo teste de Tukey.

*;** - significativo aos níveis de 5 e $1 \%$ de probabilidade, pelo teste $\mathrm{F}$.

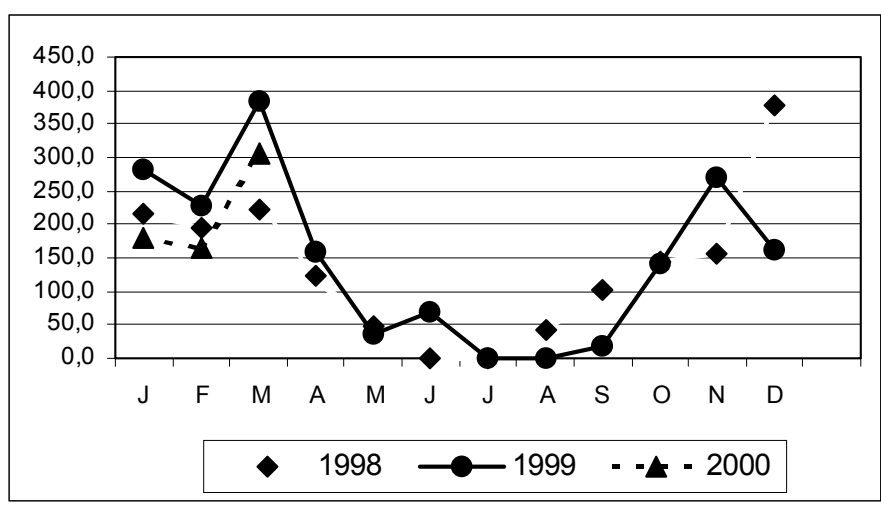

FIGURA 1 - Precipitações médias(mm) ocorridas no período de 1998 a março de $2000.9^{\circ}$ Distrito de Meteorologia de Cuiabá, Várzea Grande - MT.

o comprimento da folha 3, estão de acordo com os apresentados por Santos et al. (1982), aos 24 meses, com o PB-121 (363,20cm) e PB-111(309,00cm), nas Filipinas.

O híbrido AVeJ x GBrRN apresentou os menores comprimentos de pecíolos das folhas 3 , em todas as avaliações (Tabela 3). Esta característica, associada aos menores comprimentos da folha 3 e dos folíolos, verificados também neste híbrido, são caracteres importantes no sentido de se permitir um incremento na densidade de plantio, desde que, em futuras avaliações, estes resultados sejam confirmados.

1. O híbrido AVG x GBrPF apresenta maior circunferência do coleto, maior comprimento da folha 3 e maiores número e comprimento de folíolos da folha 3.

2. O AVeJ x GBrRN apresenta menor circunferência do coleto, comprimento de folíolos da folha3, comprimento da folha $3 \mathrm{e}$ comprimento de pecíolo da folha 3 .

3. O AAG x GBrPF apresenta maior comprimento do pecíolo.

\section{REFERÊNCIAS BIBLIOGRÁFICAS}

EMBRAPA. Centro Nacional de Pesquisa de Solos (Rio de Janeiro, RJ). Sistema brasileiro de classificação de solos. Rio de Janeiro: EMBRAPA Solos, 1999. 412p.

FERRAZ, L. G. B.; PEDROSA, A. C.; MELO, G. S. de. Avaliação do comportamento de coqueiro híbrido e cultivares nacionais. 
Recife/PE: IPA, n.5, p.1-7, 1987. (Pesquisa em andamento).

FERREIRA, J. C. V. Mato Grosso e seus municípios. Secretaria de Estado da Cultura, 1997. 668p.

OURIVER, M. Estude de la croissance et du développement du cocotier hybrid PB 121(NJM x GOA) au jeune âge. Oléagineux, Paris, v.39, n.2, p.73-80, 1984.

PERSLEY, G. J. Replanting the tree of life : towards na international agenda for coconut palm research. Wallingford: CABIACCAR, 1992. 156p.

PIRES, M. de M.; SÃO JOSÉ, A.R.; RUFINO, J.L. dos S.; BRAGA, M. J. Aspectos econômicos da cultura do coco no Brasil. In:
SÃO JOSÉ, A.R.; SOUZA, I.V.B.; MOURA, J.I.L.; REBOUÇAS, T.N.H.(Ed.). Coco: produção e mercado. Vitória da Conquista BA: DFZ/UESB, 1999. p. 7-19.

PRODUÇÃO AGRÍCOLA MUNICIPAL. Mato Grosso: IBGE, 1998.

SANTOS, G.A.; CARPIO, C.B.; ILAGAN, M.C.; CANO, S.B.; DELA CRUZ, B.V. Flowering and early yield performance of four IRHO coconuts hybrids in the Philippines. Oléagineux, Paris, v.37, n.12, p.571-580, 1982 .

SIQUEIRA, L. A. de.; SIQUEIRA, E.R.; RIBEIRO, F.E. Comportamento de híbridos de coqueiro no Nordeste do Brasil. Plantation Recherche Development, Paris, v.2, n.1, 48-53, 1995. 